by an high complexity and a considerable emotional impact. Clinical experience shows that parents are often turned away from the emergency room because they were considered an obstacle.

Objective Investigate the behaviour of some hospitals about the management of parents during cardiopulmonary resuscitation in paediatric subjects.

Materials and Methods Deliver a questionnaire to the nurse coordinators of 19 Italian hospital.

Results The questionnaire had a response rate equal to $89.4 \%$, corresponding to 17 hospitals.

$23.5 \%$ of hospitals admit the presence of both parents during all phases of resuscitation without age limits, while $17,7 \%$ of the structures do not allow the parental presence because of the possible fear created by the anxiety of parents, for the inadequacy of the spaces within the operational units and the perception that parents can potentially be an obstacle for health professionals during the resuscitation procedures.

The remaining 58.8\% have a favourable opinion about the possibility of guaranteeing to the paediatric patient, without any age limit, the presence of parents during all phases of the cardiopulmonary resuscitation but difficulties arise to make such a guarantee for the inadequacy of the spaces, for difficulties in managing the behaviour of the relatives especially in regards of anxiety and for the absence of any psychological support figure. Conclusions It's necessary that all the hospitals in any way involved into the primary care process shall act in conformity with common management protocols concerning this theme.

\section{P0-0269 CHARACTERISTICS OF CLINICAL PATHWAYS AND EMERGENCY CARE IN PAEDIATRIC SURGICAL PATHOLOGY IN LOMBARDY}

${ }^{1} \mathrm{~S}$ Maiandi, ${ }^{2} \mathrm{E}$ Gabos, ${ }^{3} \mathrm{~B}$ Cantoni. ${ }^{1}$ Pediatric Unit and Pediatric Emergency Unit, Azienda Ospedaliera Della Provincia Di Lodi, Lodi, Italy; ${ }^{2}$ Università Degli Studi Di Milano, Corso Di Laurea in Infermieristica Pediatrica, Milano, Italy; ${ }^{3}$ SITRA Area Delle Pediatrie, Fond. IRCCS Cà Granda Ospedale Maggiore Policlinico, Milano, Italy

\subsection{6/archdischild-2014-307384.923}

Background The clinical and emergency care daily activities show that only a fraction of children with urgent surgical pathology access to dedicated paediatric centres while most of the diagnostic, therapeutic and emergency care takes place at facilities not necessarily specialised in the treatment of paediatric acute illness; as it should be as per regional and national legislation.

Objective Analyse the care pathways of children hospitalised for urgent surgical pathology (appendicitis, hypertrophic pyloric stenosis).

Materials and methods Structured survey delivered to 93 hospitals in Lombardy.

Results The response rate was equal to $79.7 \%$, corresponding to 74 facilities: the appendectomy was performed in $90.5 \%$ ( $\mathrm{n}=$ 67 ) and the surgical correction of HPS was performed in $17.6 \%$ $(\mathrm{n}=13)$ of cases.

The hospitalisation was in $14.9 \%$ of cases $(n=10)$ at the paediatric surgery department, $56.7 \%(\mathrm{n}=38)$ at paediatrics department, $4.5 \%(\mathrm{n}=3)$ at general surgery department having rooms dedicated to children and $23.9 \%(n=16)$ at general surgery department without paediatrics dedicated rooms.

Discussion The analysis shows that the regional and national legislation is disregarded: it is alarming the attitude of 19 hospitals that hospitalise children in unsuitable environments, with rooms shared with adults and aged patients and the correlated risk of adopting care pathways "not dedicated and unsafe " for children.

Conclusions It's desirable an adjustment of the hospital facilities to promotes the creation of a culture in paediatrics able to ensure the highest levels of care in such a vulnerable age.

\section{PO-0270 USE OF SUCROSE AND NON-PHARMACOLOGICAL METHODS IN ALLEVIATING NEWBORN PROCEDURAL PAIN IN A PICU SETTING}

A Manou. Neonatal Medicine, Leeds Teaching Hospitals, Leeds, UK

\subsection{6/archdischild-2014-307384.924}

Background Sucrose has become standard practice across neonatal units for reducing procedural pain in preterm and term newborns. In infants admitted to PICU subject to similar procedures, systemic analgesics like morphine remains the preferred choice for procedural pain despite emerging links between these agents and adverse neurodevelopment outcomes.

Aim To explore the use of Sucrose and other non-pharmacologic methods for alleviating neonatal procedural pain in a PICU setting.

Methods Online survey sent to all medical and nursing staff in a regional PICU about use of sucrose and non-pharmacological methods for procedural pain relief in newborns.

Results There were a total of 28 respondents.

PICU staff are aware of Sucrose and non-pharmacological methods. However, they do not use these routinely for procedural pain, and $18 \%$ would never use a non-pharmacological method or sucrose.

$18 \%$ staff members do not believe these methods to be effective in controlling pain in newborns.

The reasons for not using these measures routinely were that these are unfeasible in post-operative patients (50\% respondents) and not required in patients already on morphine (7\% respondents). $25 \%$ respondents said other staff members discouraged them if they wanted to use these measures. $43 \%$ respondents want Sucrose as a part of the guideline for pain management in babies.

Conclusions Despite awareness of sucrose and non-pharmacological adjuncts, these are not consistently used in PICU. Incorporating these methods as a part of evidence based guideline for management of procedural pain can reduce the need for systemic analgesia and reduce the adverse neurodevelopmental side effects.

\section{P0-0271 A DIFFICULT DIAGNOSIS OF NATURAL KILLER CELL LYMPHOMA PRESENTING WITH BILATERAL PLEURAL EFFUSIONS}

${ }^{1}$ A Meral, ${ }^{2} S$ Kesici, ${ }^{2} \mathrm{M}$ Tanyildiz, ${ }^{3} \mathrm{Z}$ Akcoren, ${ }^{4} \mathrm{~B}$ Aydin, ${ }^{1} \mathrm{~A}$ Birbilen, ${ }^{2} \mathrm{~B}$ Bayrakci. ${ }^{1}$ Department of Pediatrics, Hacettepe University Faculty of Medicine, Ankara, Turkey; ${ }^{2}$ Department of Pediatrics Division of Pediatric Intensive Care, Hacettepe University Faculty of Medicine, Ankara, Turkey; ${ }^{3}$ Department of Pediatrics Division of Pediatric Pathology, Hacettepe University Faculty of Medicine, Ankara, Turkey; ${ }^{4}$ Department of Pediatrics Division of Pediatric Oncology, Hacettepe University Faculty of Medicine, Ankara, Turkey

\subsection{6/archdischild-2014-307384.925}

Background Natural killer (NK) cell lymphoma is a rare and aggressive neoplasm characterised by angioinvasion or angiodestruction. The aim of this report to describe a patient with an 